\title{
Microbiology Susceptibility Time Point Reference
}

National Cancer Institute

\section{Source}

National Cancer Institute. Microbiology Susceptibility Time Point Reference. NCI

Thesaurus. Code C87948.

The point in time that acts as a fixed reference point to a microbiology susceptibility assessment. 